\title{
Chaos tamed
}

\section{Even though our view of the physical world has shifted from that of determinism to randomness, randomness itself can now be exploited to retrieve a system's deterministic response.}

\begin{abstract}
Kees Wapenaar and Roel Snieder
In the nineteenth century, the world of physics was one of order. Pierre-Simon Laplace was a key proponent of the deterministic Universe. In this model, the future is completely predictable if one knows the forces between all particles as well as their positions and velocities at any one moment. Take, for instance, a ball kicked into a forest. The ball bounces repeatedly off the tree trunks, but if you know the original position of the ball, its velocity and the trees' locations, you can determine the future motion of the ball from the player's initial kick.
\end{abstract}

In the twentieth century, Heisenberg's uncertainty principle shattered the deterministic dream. In the quantum world, only the probabilities for events are constrained by the laws of quantum mechanics. So for an atom-sized soccer ball kicked into the forest, the trajectory is not determined, but the probability for every imaginable trajectory is.

Even for macroscopic systems, determinism did not survive into the twentieth century. At that point Henri Poincaré, in a visionary anticipation of chaos theory, showed that even tiny uncertainties in initial conditions can grow exponentially with time to make motion at a later time indeterminable, for all practical purposes. So, when the soccer ball is kicked a number of times in slightly different directions, it hits the same trees during the first few bounces at slightly different positions; but over time the trajectories diverge, and after a few bounces the ball may move in completely different ways between the trees.

So much for particles: waves behave completely differently. If a referee blows her whistle in the same forest repeatedly at slightly different positions, the sound waves scattering between the trees change much less than the motion of the ball. One reason is that waves have an intrinsic length scale, the wavelength, and any perturbations affecting the waves over this length scale are effectively smoothed out.

Recent research has shown that acoustic noise can be used to synthesize deterministic waves generated by a point source - like the whistle-blowing referee. Imagine it's raining in the forest. Every raindrop excites acoustic waves that bounce among the trees in an apparently random fashion.

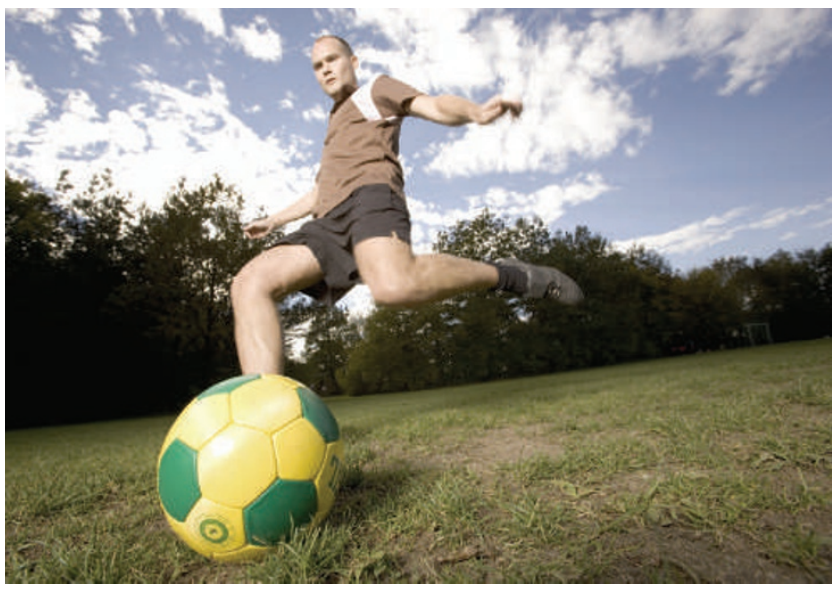

in thermal equilibrium, the deterministic response of the system is related to thermal fluctuations. This principle can be extended to systems so large that thermal fluctuations are irrelevant, such as the sound waves generated by raindrops falling in the forest. It has recently been shown theoretically that the principle holds for a wide class of linear

Nevertheless, the trees leave an imprint on the wave field that is characteristic for the forest. The unravelling of this imprint turns out to be surprisingly simple. Let's say that, instead of a whistle-blowing referee, there are two microphones in the forest. With a standard computer operation - crosscorrelation - we can reconstruct the sound of the referee's whistle from the recorded noise of falling raindrops.

Take one raindrop that falls in line with the two microphones. The sound wave it generates travels forward, reaching the nearest microphone first and then continuing to the farther one. The difference in the time it takes for the wave to reach each microphone equals the time it takes for the wave to travel between the two microphones. The cross-correlation of the sound waves recorded by the microphones produces a signal at precisely this travel time. So it is as if the first microphone acts as a source, transmitting a weak sound wave to the second. This is enhanced by other raindrops falling in line with the microphones; the rest of the raindrops do not produce a coherent signal. Taking all the coherent waves together, the first microphone acts as if it is transmitting the sound of the whistle to the second. The reproduced sound can be used for imaging - say, to determine the position of nearby trees. This principle presents the opportunity to do wave experiments without using active sources.

It has been known since Albert Einstein's seminal 1905 paper on brownian motion that the diffusion of a particle is related to the way it slows down when it is disturbed in some way. This principle was later generalized to the fluctuation-dissipation theorem, which states that for systems systems, including electromagnetism, flowing media and quantum mechanics.

Extracting the deterministic response of a system from noise is amazing enough, but there is more. According to theory, noise sources must be distributed homogeneously throughout space, and be uncorrelated. That is, the raindrops must fall everywhere in the forest, and fall at statistically independent times and locations. Astonishingly, in many applications the extraction of the system response from noise is fairly robust when noise sources are limited and irregularly distributed, probably because of the stability of wave propagation.

Our view of the Universe may have shifted from the deterministic to the random, but since the turn of the last century physics itself has provided a less simplistic view. Fields generated by random sources can be used for imaging and for monitoring of systems such as Earth's subsurface, or of mechanical structures such as bridges. Randomness is no longer at odds with determinism, it has instead become a new window on the deterministic response of the physical world.

Kees Wapenaar is in the Department of Geotechnology, Delft University of Technology, PO Box 5048, 2600 GA

Delft, the Netherlands.

Roel Snieder is at the Center for Wave Phenomena, Colorado School of Mines, Golden, Colorado 8041-1887, USA.

FURTHER READING

Berry, M. V. Proc. R. Soc. Lond. A 413, 183-198 (1987). Snieder, R. K. \& Scales, J. A. Phys. Rev. E 58, 5668-5675 (1998).

Snieder, R., Wapenaar, K. \& Wegler, U. Phys. Rev. E 75, 036103 (2007).

Weaver, R. L. Science 307, 1568 (2005). 